\title{
Negative regulation of ERK1/2 by PI3K is required for the protective effects of Pyropia yezoensis peptide against perfluorooctane sulfonate-induced endoplasmic reticulum stress
}

\author{
JEONG HWAN OH${ }^{1}$, EUN-YOUNG KIM ${ }^{1}$, YOUN HEE CHOI ${ }^{2}$ and TAEK-JEONG NAM ${ }^{1,3}$ \\ ${ }^{1}$ Cell Biology Laboratory, Institute of Fisheries Sciences, Pukyong National University, Busan 46041; \\ Departments of ${ }^{2}$ Marine Bio-Materials and Aquaculture, and ${ }^{3}$ FoodScience and Nutrition, \\ Pukyong National University, Busan 48513, Republic of Korea
}

Received November 3, 2016; Accepted January 19, 2017

DOI: $10.3892 / \mathrm{mmr} .2017 .6285$

\begin{abstract}
Perfluorooctane sulfonate (PFOS) is a stable fluorosurfactant, which causes adverse effects in various organisms. The present study was designed to investigate the effects of Pyropia yezoensis peptide (PYP), a peptide comprised of 11 residues (ALEGGKSSGGG), on PFOS-induced endoplasmic reticulum (ER) stress in Chang cells. PFOS exposure $(400 \mu \mathrm{M})$ for $24 \mathrm{~h}$ significantly decreased cell viability, which was upregulated by $250-1,000 \mathrm{pg} / \mathrm{ml}$ PYP treatment. Exposure to PFOS also significantly increased expression of the ER stress response protein, glucose-regulated protein 78 (GRP78), and phosphorylation of extracellular signal-regulated kinase $1 / 2$ (ERK1/2). These elevations were significantly decreased by PYP (250 pg/ml), and, in particular, the PFOS-induced GRP78 upregulation was decreased following treatment with $10 \mu \mathrm{M}$ SL327, an ERK-kinase inhibitor. However, PYP-induced decreases in GRP78 expression and ERK1/2 phosphorylation were upregulated following treatment with LY294002 $(20 \mu \mathrm{M})$, a phosphatidylinositol-3 kinase (PI3K) inhibitor. PFOS-induced apoptosis was also significantly attenuated by PYP $(250 \mathrm{pg} / \mathrm{ml})$ treatment, and the PYP-induced reduction in apoptosis was abolished by inhibition of PI3K. These findings indicate that negative regulation of ERK1/2 by $\mathrm{PI} 3 \mathrm{~K}$ is essential for the protective effects of PYP against PFOS-induced cell death, suggesting that PYP may be a candidate for therapeutic use.
\end{abstract}

\section{Introduction}

Perfluorooctane sulfonate (PFOS) is an organofluorine compound and a synthetic, stable fluorosurfactant that is

Correspondence to: Professor Taek-Jeong Nam, Cell Biology Laboratory, Institute of Fisheries Sciences, Pukyong National University, 474 Ilgwang-ro, Ilgwang-myeon, Gijang-gun, Busan 46041, Republic of Korea

E-mail: namtj@pknu.ac.kr

Key words: endoplasmic reticulum stress, Pyropia yezoensis peptide, perfluorooctane sulfonate used as a surface protector for paper, food containers, carpets and various other applications due to its hydrophobic and lipophobic properties (1). Fluorine has the highest electronegativity in fluorocarbons, resulting in formation of a strong carbon-fluorine (C-F) covalent bond, thus inducing resistance to hydrolysis, photolysis and biodegradation. Therefore, fluorocarbons are considered persistent organic pollutants, and pharmacokinetic studies on PFOS have been conducted in fish, monkeys, chickens and humans (2-4). These studies revealed that PFOS has a relatively long depuration half-life, which may disturb cellular function. Although the mechanisms underlying the toxicity of PFOS have not been fully established, the chemical is known to induce oxidative stress and cellular damage, including hepatocellular hypertrophy and the inhibition of intracellular communication $(5,6)$.

The endoplasmic reticulum (ER) is a major organelle that is involved in protein modification and folding, as well as intracellular calcium homeostasis. Cellular stress-induced protein damage and alteration of redox status results in a reduction of folding capacity and the accumulation of misfolded proteins in the ER lumen, which activates a series of signaling pathways known as the ER stress response $(7,8)$. Glucose-regulated protein 78 (GRP78), which is an ER stress sensor, is an ATP-dependent protein chaperone localized in the ER lumen. Under ER stress, GRP78 binds unfolded proteins and activates a multi-chaperone complex, resulting in increased ER protein folding capacity (9). However, severe and long-lasting ER stress results in the accumulation of unfolded or misfolded proteins and subsequent cell death.

Pyropia yezoensis is a red alga that has been cultured as food and a nutritional supplement due to its biofunctional components, including proteins, vitamins, minerals and mycosporine-like amino acids (10). In particular, $P$. yezoensis peptide (PYP) is known to have antioxidant and chemoprotective properties $(11,12)$. However, the bioactivity of PYP in ER stress conditions induced by environmental pollutants has yet to be elucidated.

The present study was designed to investigate the hypothesis that the protective effects of PYP against PFOS exposure are associated with the ER stress response, and that this is mediated by the phosphatidylinositol-3 kinase (PI3K) and 
extracellular signal-regulated kinase 1/2 (ERK1/2) signal pathways. To investigate this hypothesis, it was determined whether i) pretreatment with PYP decreases ER stress caused by PFOS exposure; ii) the PYP-induced decrease in PFOS-induced ER stress is associated with the PI3K and ERK1/2 signaling pathways, and iii) apoptosis induced by PFOS exposure is regulated by PYP-induced activation of the PI3K signaling pathway.

\section{Materials and methods}

Cell culture and chemicals. Chang cells were purchased from American Type Culture Collection (Manassas, VA, USA; cat. no. CCL-13). This cells line is known to have been contaminated with HeLa cervical adenocarcinoma cells. The cells were cultured in minimum essential medium containing non-essential amino acids (Sigma-Aldrich; Merck KGaA, Darmstadt, Germany), $1 \mathrm{mM}$ sodium pyruvate, $10 \%$ fetal bovine serum (GenDEPOT, Inc., Barker, TX, USA), $100 \mathrm{U} / \mathrm{ml}$ penicillin and $100 \mathrm{mg} / \mathrm{ml}$ streptomycin at $37^{\circ} \mathrm{C}$ in a humidified incubator containing 5\% $\mathrm{CO}_{2}$. PFOS (cat. no. 2795-39-3; $>98 \%$ ) and dimethyl sulfoxide (DMSO; cat. no. 67-68-5; $>99.9 \%$ ) were purchased from Sigma-Aldrich; Merck KGaA, and LY294002 (cat. no. 1130) and SL327 (cat. no. 1969) were obtained from Tocris Bioscience (Bristol, UK). PFOS and inhibitors were dissolved in DMSO. The minimal concentration of DMSO $(<0.001 \%)$ was used to prevent cellular damage.

Cell viability assay. Cell viability was determined using Cyto- ${ }^{\mathrm{TM}}$ cell viability assay kit (LPS Solution, Daejeon, South Korea). Cells were seeded at a density of $1 \times 10^{4}$ cells/well in a 96-well plate (final volume, $100 \mu \mathrm{l} / \mathrm{well}$ ), and were incubated for $24 \mathrm{~h}$ at $37^{\circ} \mathrm{C}$ in a humidified incubator containing $5 \% \mathrm{CO}_{2}$. Cells were then exposed to PFOS $(25-400 \mu \mathrm{M})$ for $24 \mathrm{~h}$, with or without pretreatment with PYP $(62.5,125$, 250,500 or $1,000 \mathrm{pg} / \mathrm{ml}$ ) for $2 \mathrm{~h}$ at $37^{\circ} \mathrm{C}$. PYP is a peptide comprising 11 residues (ALEGGKSSGGG), which was synthesized by Peptron (Daejeon, South Korea) according to a previous study (12). Subsequently, a water-soluble tetrazolium salt $(10 \mu \mathrm{l} /$ well $)$ was added and the cells were incubated for $30 \mathrm{~min}$ at $37^{\circ} \mathrm{C}$ in a $5 \% \mathrm{CO}_{2}$ incubator. Colored formazan was measured by examining the absorbance at $450 \mathrm{~nm}$.

Western blotting. PFOS-induced ER stress was confirmed using immunoblotting. Cells were treated with PFOS $(400 \mu \mathrm{M})$ for $24 \mathrm{~h}$ at $37^{\circ} \mathrm{C}$, with or without PYP $(250 \mathrm{pg} / \mathrm{ml})$ pretreatment for $2 \mathrm{~h}$ at $37^{\circ} \mathrm{C}$. Additionally, 1 group was treated with PYP $(250 \mathrm{pg} / \mathrm{ml})$ only. SL327 $(10 \mu \mathrm{M})$ and LY294002 $(20 \mu \mathrm{M})$ treatment was performed for $30 \mathrm{~min}$ prior to the PYP pretreatment. Following treatment, the media was removed and cells were washed twice with $1 \mathrm{X}$ PBS. Cell lysis was performed with radioimmunoprecipitation assay buffer containing a protease inhibitor cocktail (Calbiochem; Merck $\mathrm{KGaA}$ ). Protein concentrations were determined using a bicinchoninic acid protein assay kit (Thermo Fisher Scientific, Inc., Waltham, MA, USA), and proteins ( $20 \mu \mathrm{g})$ were separated by $15 \%$ SDS-polyacrylamide gel electrophoresis. The separated proteins were transferred to a polyvinylidene fluoride membrane. The membrane was blocked with a blocking buffer containing $1 \%$ bovine serum albumin (GenDEPOT, Inc.) in
TBST (TBS containing 0.1\% Tween-20) and was then probed with the following primary antibodies: Anti-GRP78 (1:1,000; cat. no. ab108613; Abcam, Cambridge, UK), anti-pERK1/2 (1:1,000; cat. no. sc-7383; Santa Cruz Biotechnology, Inc., Dallas, TX, USA), ERK1 (1:1,000; cat. no. sc-94; Santa Cruz Biotechnology, Inc.), ERK2 (1:1,000; cat. no. sc-154; Santa Cruz Biotechnology, Inc.) and anti- $\beta$-actin $(1: 1,000$; cat. no. sc-47778; Santa Cruz Biotechnology, Inc.) overnight at $4^{\circ} \mathrm{C}$ with agitation. Following washing three times with TBST for $10 \mathrm{~min}$, the membrane was incubated with the appropriate secondary antibody (cat. no. 31460; Thermo Fisher Scientific, Inc.) at a dilution of 1:10,000 for $1 \mathrm{~h}$ at room temperature. b-actin was probed for the normalization of blots.

Detection of apoptotic cells. Cells were treated with $400 \mu \mathrm{M}$ PFOS for $24 \mathrm{~h}$ at $37^{\circ} \mathrm{C}$, with or without PYP pretreatment $\left(250 \mathrm{pg} / \mathrm{ml}\right.$ ) for $2 \mathrm{~h}$ at $37^{\circ} \mathrm{C}$. Apoptosis was measured using the Muse Annexin V and Dead Cell Assay kit (Merck KGaA) according to the manufacturer's protocol. Annexin V staining was measured by Muse ${ }^{\circledast}$ Cell Analyzer and the data was analysed by Muse 1.3.1 software (Merck KGaA).

Statistical analysis. All experiments were performed in triplicate and the data were expressed as the mean \pm standard error of the mean for each group. For western blotting, the difference in expression levels was determined by densitometric analysis, using ImageJ 1.48 software (National Institutes of Health, Bethesda, MD, USA). Data were statistically analyzed by one-way analysis of variance followed by Tukey's multiple comparison tests using GraphPad Prism 5 software (GraphPad Software, Inc., La Jolla, CA, USA). P $<0.05$ was considered to indicate a statistically significant difference.

\section{Results}

PYP attenuates PFOS-induced decreases in cell viability and downregulates PFOS-induced increases in GRP78 expression in Chang cells. To investigate the effects of PYP on PFOS-induced cell damage, the optimal dose of PFOS was initially determined based on a cell viability assay. Exposure to PFOS (100 and $400 \mu \mathrm{M})$ significantly decreased the viability of Chang cells compared with the untreated control cells (Fig. 1A). Following exposure to $400 \mu \mathrm{M}$ PFOS for $24 \mathrm{~h}$ with or without PYP pretreatment $(62.5-1,000 \mathrm{pg} / \mathrm{ml})$, cell viability was assessed in a parallel manner. Pretreatment with PYP $(250-1,000 \mathrm{pg} / \mathrm{ml})$ significantly attenuated the PFOS-induced decrease in cell viability compared with in cells exposed to PFOS alone (Fig. 1B). To further investigate whether the PYP-induced increase in cell viability was associated with the ER stress response, cell damage was induced by $400 \mu \mathrm{M}$ PFOS in order to decrease the viability of Chang cells by $\sim 50 \%$. Subsequently, ER stress was assessed by measuring the expression levels of GRP78 by western blotting. GRP78 expression was upregulated following exposure to PFOS for $24 \mathrm{~h}$ compared with the control cells; however, GRP78 expression was significantly downregulated by pretreatment with PYP $(250 \mathrm{pg} / \mathrm{ml}$; Fig. 1C). There was no significant alteration in GRP78 expression between the control group and the group treated with PYP alone (250 pg/ml; Fig. 1C). 
A

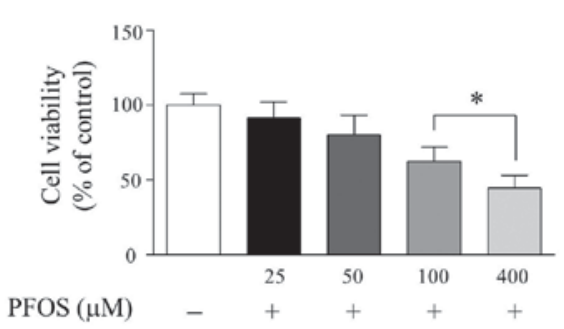

B

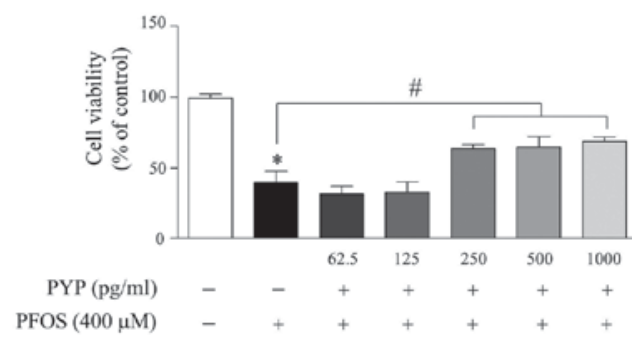

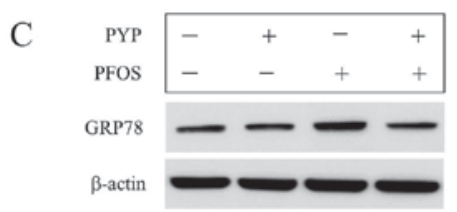

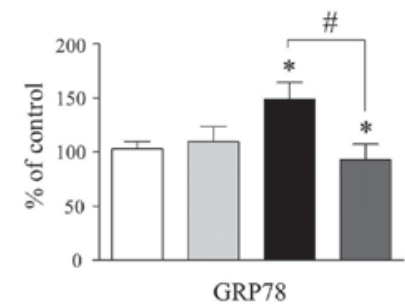

Figure 1. Effects of PYP treatment on cell viability and PFOS-induced ER stress. (A) PFOS decreased the viability of Chang cells in a dose-dependent manner. (B) Pretreatment with PYP (250-1,000 pg/ml) significantly increased the viability of PFOS-treated cells. (C) Exposure to PFOS (400 $\mu$ M) significantly upregulated GRP78 expression, which was attenuated by pretreatment with PYP $(250 \mathrm{pg} / \mathrm{ml})$. ${ }^{*} \mathrm{P}<0.05$ vs. control group; ${ }^{*} \mathrm{P}<0.05$ vs. $\mathrm{PFOS}(400 \mu \mathrm{M})$ exposure. PYP, Pyropia yezoensis peptide; ER, endoplasmic reticulum; PFOS, perfluorooctane sulfonate; GRP78, glucose-regulated protein 78.

A
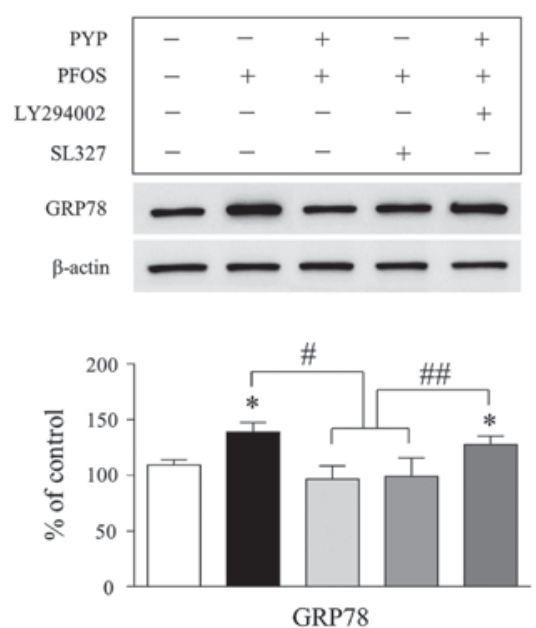

B
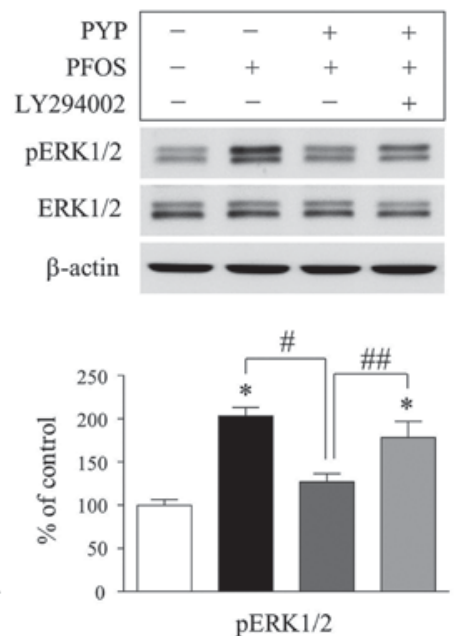

Figure 2. PYP induced activation of PI3K-dependent signaling and decreased ERK1/2-mediated ER stress in response to PFOS exposure. (A) PFOS-induced increase in GRP78 expression was significantly downregulated by pretreatment with SL327 (10 $\mu$ M). Inhibition of PI3K with LY294002 (20 $\mu$ M) attenuated the $250 \mathrm{pg} / \mathrm{ml}$ PYP- and SL327-induced decrease in GRP78 expression. (B) Phosphorylation of ERK1/2 by PFOS (400 $\mu \mathrm{M})$ was decreased by PYP (250 pg/ml) pretreatment, which was upregulated by inhibition of $\mathrm{PI} 3 \mathrm{~K}$. ${ }^{*} \mathrm{P}<0.05$ vs. control group; ${ }^{\#} \mathrm{P}<0.05$ vs. $\mathrm{PFOS}(400 \mu \mathrm{M})$ exposure; ${ }^{\# \prime} \mathrm{P}<0.05$ vs. pretreatment with PYP or SL327. PYP, Pyropia yezoensis peptide; PI3K, phosphatidylinositol-3 kinase; ERK1/2, extracellular signal-regulated kinase 1/2; ER, endoplasmic reticulum; PFOS, perfluorooctane sulfonate; GRP78, glucose-regulated protein 78 .

Inhibition of PI3K attenuates PYP-induced decreases in GRP78 expression and ERK1/2 phosphorylation by PFOS. To investigate the intracellular events modulated by PYP against PFOS-induced ER stress, the present study aimed to determine i) whether PFOS-induced GRP78 expression was associated with the PI3K and ERK1/2 signal pathways, and ii) whether there was an interaction between PI3K and ERK1/2 for PYP-induced decreases in PFOS-induced ER stress. Pretreatment with SL327 $(10 \mu \mathrm{M})$, an ERK-kinase inhibitor, significantly downregulated PFOS-induced GRP78 expression compared with cells exposed to PFOS alone. Conversely, pretreatment with LY294002 (20 $\mu \mathrm{M})$, an inhibitor of PI3K, upregulated $250 \mathrm{pg} / \mathrm{ml}$ PYP-inhibited GRP78 expression compared with cells exposed to PFOS and pretreated with PYP (Fig. 2A). Since activation of PI3K-dependent signalling (as inhibition of PI3K attenuated the PYP-induced decrease in PFOS-induced ER stress) and inhibition of ERK1/2 phosphorylation were associated with PYP-induced decreases in PFOS-induced ER stress, the present study aimed to determine whether PYP-induced activation of PI3K regulated phosphorylation of ERK1/2. Phosphorylation of ERK $1 / 2$ by PFOS was significantly 

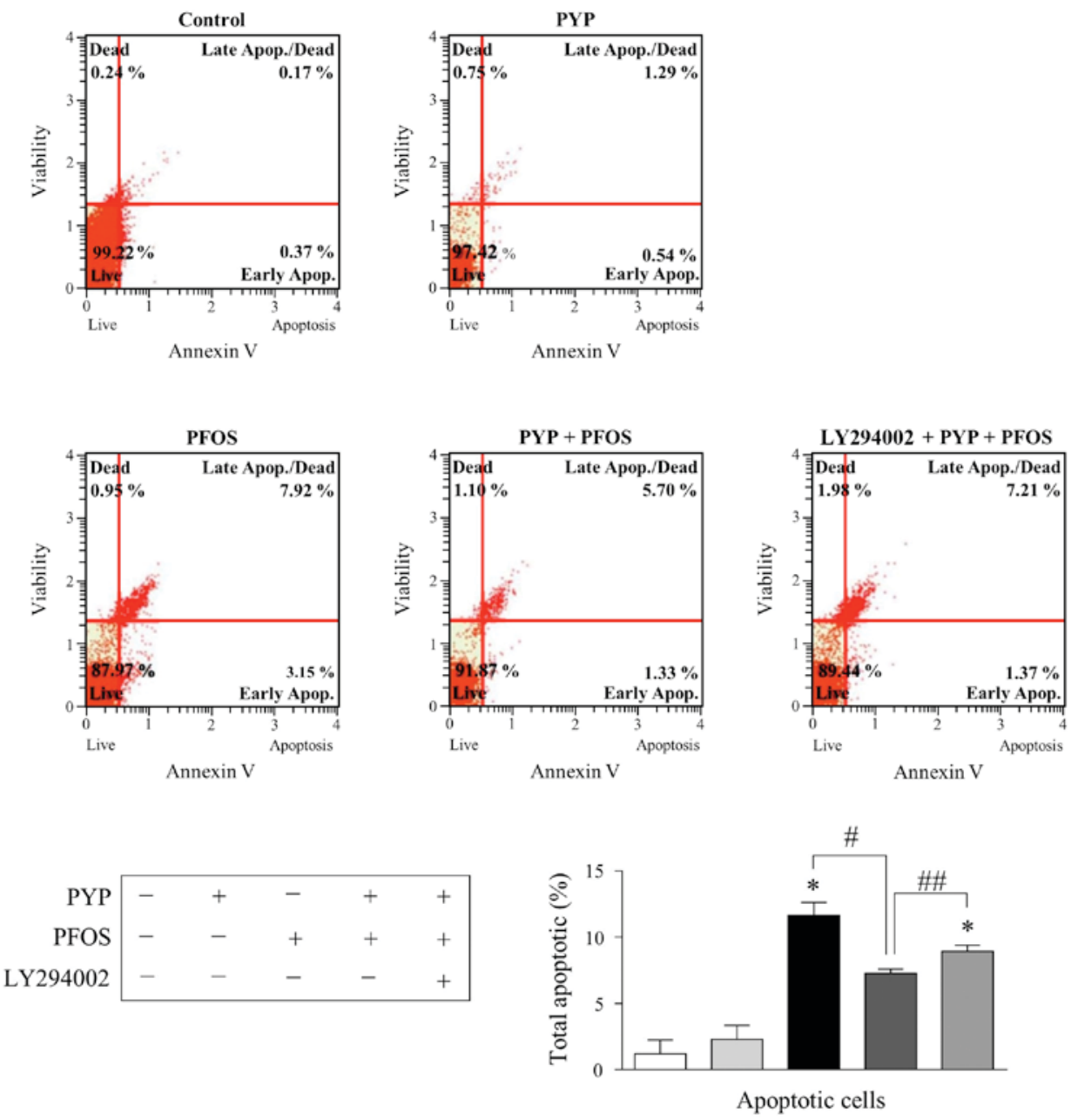

Figure 3. Protective effects of PYP against PFOS-induced cell death. Exposure to PFOS (400 $\mu \mathrm{M})$ increased the rate of apoptosis, which was significantly attenuated by pretreatment with PYP $(250 \mathrm{pg} / \mathrm{ml})$. The PYP-induced decrease in apoptosis was attenuated by inhibition of phosphatidylinositol-3 kinase with LY294002 $(20 \mu \mathrm{M}) .{ }^{*} \mathrm{P}<0.05$ vs. control group; ${ }^{\#} \mathrm{P}<0.05$ vs. PFOS $(400 \mu \mathrm{M})$ exposure; ${ }^{\# \#} \mathrm{P}<0.05$ vs. LY294002 pretreatment. PYP, Pyropia yezoensis peptide; PFOS, perfluorooctane sulfonate.

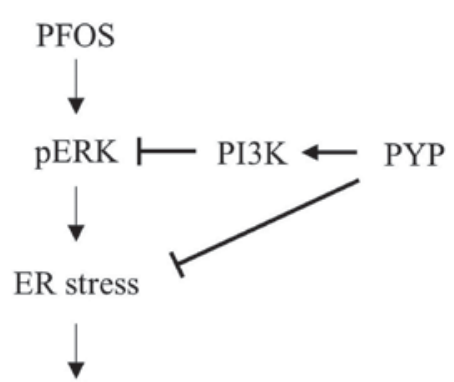

Cell death

Figure 4. Schematic of a proposed mechanism underlying the protective effects of PYP against PFOS exposure in Chang cells. PFOS-induced ER stress is mediated by phosphorylation of ERK1/2. The phosphorylation of ERK1/2 and ER stress are downregulated by PYP-induced activation of PI3K. PYP increases the viability of Chang cells via a decrease in PFOS-induced ER stress by PI3K activation, attenuating PFOS-induced apoptosis. PYP, Pyropia yezoensis peptide; PFOS, perfluorooctane sulfonate; ER, endoplasmic reticulum; ERK1/2, extracellular signal-regulated kinase 1/2; PI3K, phosphatidylinositol-3 kinase.

decreased by PYP $(250 \mathrm{pg} / \mathrm{ml})$ treatment, which was abolished by inhibition of PI3K with LY294002 (20 $\mu$ M; Fig. 2B).
There was no significant difference in the total ERK1/2 expression compared with the control (Fig. 2B).

PFOS-induced cell death was decreased by activation of PI3K by PYP treatment. Since PYP treatment enhanced cell viability and decreased PFOS-induced ER stress, it was determined whether PFOS-induced apoptosis was regulated by PYP treatment. The number of apoptotic cells was significantly increased by PFOS $(400 \mu \mathrm{M})$ exposure for $24 \mathrm{~h}$ compared with the control cells (Fig. 3). Pretreatment with PYP $(250 \mathrm{pg} / \mathrm{ml})$ significantly decreased PFOS-induced apoptosis; however, this effect was attenuated by inhibition of PI3K with LY294002 (20 $\mu \mathrm{M}$; Fig. 3A). There was no significant difference between the PYP and control groups.

\section{Discussion}

The present study was performed to investigate the protective effects of PYP against PFOS-induced ER stress in Chang cells. PYP $(250 \mathrm{pg} / \mathrm{ml})$ treatment downregulated the PFOS-induced increase in ER stress, mediated by phosphorylation of ERK1/2. The PYP-induced decrease in ER stress was reversed by inhibition of PI3K. In addition, PFOS-induced 
apoptosis was significantly decreased by PYP treatment, which was abolished by inhibition of PI3K. Therefore, these findings demonstrated that the protective effects of PYP occur via inhibition of ERK1/2, resulting in the activation of PI3K against PFOS-induced ER stress.

Persistent organic pollutants, such as PFOS, induce oxidative stress, which induces apoptosis and developmental and reproductive toxicity in rats and freshwater fish. In particular, PFOS-induced oxidative stress results in ER stress and affects intracellular signaling, which subsequently leads to cell death (13-17). In the present study, it was demonstrated that pretreatment with PYP $(250-1,000 \mathrm{pg} / \mathrm{ml})$ enhanced the viability of Chang cells against PFOS $(400 \mu \mathrm{M})$ exposure, and PYP $(250 \mathrm{pg} / \mathrm{ml})$ treatment significantly decreased ER stress by PFOS (400 $\mu \mathrm{M})$ (Fig. 1). These results suggested that PYP may protect Chang cells against the ER stress caused by PFOS exposure.

To further explore the mechanisms underlying the inhibitory effects of PYP on PFOS-induced ER stress, PI3K and ERK1/2 signal pathways were assessed by western blotting. PI3K and ERK signaling pathways are known to be involved in controlling cell survival and proliferation in response to various extracellular stimuli $(18,19)$. However, PI3K and ERK1/2 inhibit each other and negatively regulate the other's pathway $(20,21)$. Although ERK1/2 has been demonstrated to promote cell survival, activation of ERK1/2 also induces apoptosis depending on the cell type examined $(22,23)$. In the present study, the PFOS-induced increase in GRP78 expression was downregulated by PYP (250 pg/ml) treatment, as well as following inhibition of ERK1/2 with SL327 $(10 \mu \mathrm{M})$. However, the PYP-induced decrease in GRP78 expression was upregulated upon inhibition of PI3K with LY294002 (20 $\mu \mathrm{M}$; Fig. 2A). Phosphorylation of ERK1/2 by PFOS (400 $\mu \mathrm{M})$ exposure was also decreased by PYP $(250 \mathrm{pg} / \mathrm{ml})$ treatment, which was significantly upregulated by inhibition of PI3K (Fig. 2B). In addition, PFOS-induced apoptosis was significantly decreased by PYP $(250 \mathrm{pg} / \mathrm{ml})$ treatment, and was abolished following inhibition of PI3K (Fig. 3).

In conclusion, these results demonstrated that i) PFOS-induced ER stress is mediated by phosphorylation of ERK1/2; ii) ERK1/2 phosphorylation is negatively regulated by PYP-induced activation of PI3K; and iii) inhibition of PI3K reverses the PYP-induced decrease in PFOS-induced ER stress and apoptosis (Fig. 4). These findings suggested that negative regulation of ERK1/2 by $\mathrm{PI} 3 \mathrm{~K}$ is essential for the protective effects of PYP against PFOS-induced ER stress-induced apoptosis of Chang cells. Thus, PYP may be used to decrease the health risks caused by environmental pollutants.

\section{Acknowledgements}

The present study was supported by the Basic Science Research Program through the National Research Foundation of Korea, funded by the Ministry of Education (grant no. 2012R1A6A1028677).

\section{References}

1. Lemal DM: Perspective on fluorocarbon chemistry. J Org Chem 69: 1-11, 2004.
2. Kannan K, Tao L, Sinclair E, Pastva SD, Jude DJ and Giesy JP: Perfluorinated compounds in aquatic organisms at various trophic levels in a Great Lakes food chain. Arch Environ Contam Toxicol 48: 559-566, 2005.

3. Loccisano AE, Campbell JL Jr, Andersen ME and Clewell HJ III: Evaluation and prediction of pharmacokinetics of PFOA and PFOS in the monkey and human using a PBPK model. Regul Toxicol Pharmacol 59: 157-175, 2011.

4. Yoo H, Guruge KS, Yamanaka N, Sato C, Mikami O, Miyazaki S, Yamashita N and Giesy JP: Depuration kinetics and tissue disposition of PFOA and PFOS in white leghorn chickens (Gallus gallus) administered by subcutaneous implantation. Ecotoxicol Environ Saf 72: 26-36, 2009.

5. Hu W, Jones PD, Upham BL, Trosko JE, Lau C and Giesy JP: Inhibition of gap junctional intercellular communication by perfluorinated compounds in rat liver and dolphin kidney epithelial cell lines in vitro and Sprague-Dawley rats in vivo. Toxicol Sci 68: 429-436, 2002.

6. Hu XZ and Hu DC: Effects of perfluorooctanoate and perfluorooctane sulfonate exposure on hepatoma Hep G2 cells. Arch Toxicol 83: 851-861, 2009.

7. Zeeshan HM, Lee GH, Kim HR and Chae HJ: Endoplasmic reticulum stress and associated ROS. Int J Mol Med 17: 327, 2016.

8. Darling NJ and Cook SJ: The role of MAPK signalling pathways in the response to endoplasmic reticulum stress. Biochim Biophys Acta 1843: 2150-2163, 2014.

9. Gardner BM, Pincus D, Gotthardt K, Gallagher CM and Walter P: Endoplasmic reticulum stress sensing in the unfolded protein response. Cold Spring Harb Perspect Biol 5: a013169, 2013.

10. MacArtain P, Gill CI, Brooks M, Campbell R and Rowland IR: Nutritional value of edible seaweeds. Nutr Rev 65: 535-543, 2007.

11. Cao J, Wang J, Wang S and Xu X: Porphyra Species: A mini-review of its pharmacological and nutritional properties. J Med Food 19: 111-119, 2016.

12. Choi YH, Kim EY, Mikami K and Nam TJ: Chemoprotective effects of a recombinant protein from Pyropia yezoensis and synthetic peptide against acetaminophen-induced Chang liver cell death. Int J Mol Med 36: 369-376, 2015.

13. Ankley GT, Kuehl DW, Kahl MD, Jensen KM, Linnum A, Leino RL and Villeneuve DA: Reproductive and developmental toxicity and bioconcentration of perfluorooctanesulfonate in a partial life-cycle test with the fathead minnow (Pimephales promelas). Environ Toxicol Chem 24: 2316-2324, 2005.

14. Chen T, Zhang L, Yue JQ, Lv ZQ, Xia W, Wan YJ, Li YY and Xu SQ: Prenatal PFOS exposure induces oxidative stress and apoptosis in the lung of rat off-spring. Reprod Toxicol 33: 538-545, 2012.

15. Liu C, Yu K, Shi X, Wang J, Lam PK, Wu RS and Zhou B: Induction of oxidative stress and apoptosis by PFOS and PFOA in primary cultured hepatocytes of freshwater tilapia (Oreochromis niloticus). Aquat Toxicol 82: 135-143, 2007.

16. Oakes KD, Sibley PK, Martin JW, MacLean DD, Solomon KR, Mabury SA and Van Der Kraak GJ: Short-term exposures of fish to perfluorooctane sulfonate: Acute effects on fatty acyl-CoA oxidase activity, oxidative stress, and circulating sex steroids. Environ Toxicol Chem 24: 1172-1181, 2005.

17. Shi $X$ and Zhou B: The role of Nrf2 and MAPK pathways in PFOS-induced oxidative stress in zebrafish embryos. Toxicol Sci 115: 391-400, 2010.

18. Engelman JA, Luo J and Cantley LC: The evolution of phosphatidylinositol 3-kinases as regulators of growth and metabolism. Nat Rev Genet 7: 606-619, 2006.

19. McCubrey JA, Steelman LS, Chappell WH, Abrams SL, Wong EW, Chang F,Lehmann B, Terrian DM, Milella M, Tafuri A, et al: Roles of the Raf/MEK/ERK pathway in cell growth, malignant transformation and drug resistance. Biochim Biophys Acta 1773: 1263-1284, 2007.

20. Mendoza MC,Er EE and Blenis J: The Ras-ERK and PI3K-mTOR pathways: Cross-talk and compensation. Trends Biochem Sci 36: 320-328, 2011.

21. Dai R, Chen $R$ and Li H: Cross-talk between PI3K/Akt and MEK/ERK pathways mediates endoplasmic reticulum stress-induced cell cycle progression and cell death in human hepatocellular carcinoma cells. Int J Oncol 34: 1749-1757, 2009.

22. Garg TK and Chang JY: Oxidative stress causes ERK phosphorylation and cell death in cultured retinal pigment epithelium: Prevention of cell death by AG126 and 15-deoxy-delta 12 , 14-PGJ2. BMC Ophthalmol 3: 5, 2003.

23. Tang D, Wu D, Hirao A, Lahti JM, Liu L, Mazza B, Kidd VJ, Mak TW and Ingram AJ: ERK activation mediates cell cycle arrest and apoptosis after DNA damage independently of p53. J Biol Chem 277: 12710-12717, 2002. 